\title{
Thai Traditional Midwives (Moh Tum Yae) in Caring for Mothers and Infants, in Petchaburi Province, Thailand: Case Studies
}

\author{
Angsinee Kansukcharearn
}

\begin{abstract}
Thai Traditional Midwives who practiced caring for pregnant women and infants in Thailand will be fade-out from Thai society but their Thai Traditional Midwives wisdom is still dominant in contemporary times and are perpetuated by closed female family relatives. The purpose of this study were to study and collect the local wisdom of Thai Traditional Midwives in Thailand. A qualitative research design was used. Eight Thai Traditional Midwives were interviewed. Data from in-depth interviews was analyzed by the process of manifest content analysis. The finding have shown that two thematic meanings of experiences of folk medical Thai midwifery wisdom in Thailand emerged from the data. They were "Belief in caring mothers and infants" and "Thai midwifery ceremonies". The first theme was represented by two categories: "a religious ceremonies" and "regarding to holy objects and spirits. The second theme was represented by Thai midwifery in antenatal period ceremonies and postnatal period ceremonies. The majority of Thai mothers who adhered to traditional postpartum practices were able to relate the notion of regaining heat and herbs. The belief of Traditional midwives is strongly influenced by Buddhism, Brahmanism and Spirits. Their belief can influence childbirth and human life. This reflects a deep understanding and integration of Buddhist doctrine in the client.
\end{abstract}

Index Terms - Thai traditional midwife, self caring, mothers and infants.

\section{INTRODUCTION}

Maternity and child health are both critical concepts in the discipline of Thai midwifery. In Thailand the modern midwifery act has replaced the term 'Traditional Midwifery'. Although Thai Traditional Midwives or "Moh Tum Yae"who practiced caring for pregnant women and infants in Thailand will be fade-out from Thai society, the wisdom of Thai Traditional Midwives is still dominant in contemporary times and are perpetuated by closed female family relatives [1]. Wisdom is defined by Walshas "the deep understanding and practice of skills to matters that are central issues of life" [2]. Furthermore, Buddhadasa defined wisdom as"correct knowledge, and understanding of the true nature of things which connect human being to the peper way with their most appropriate environment" [3]. To live with wisdom is to know relateto things by understanding their process, cause and effect.Creating thesedesired results can only be done by understanding the cause of the problem. The way to address the problem depends on one's respective needs [4]. Petchaburi Province is located in the South - western regionof

Manuscript received August 24, 2013; revised October 25, 2013.

Angsinee Kansukcharearn is with the Prachomklao College of Nursing, Thailand (e-mail: ang_sinee@yahoo.com).
Thailand. This site are wooded areas and mountain complexs so this terrain has an effect on some of the areas district, along distance away from health care centers, especially KaengKrachan and Nong Ya Plong district. It can be found that Thai Traditional Midwifery is still practiced in maternal and child health.Midwivesare people who have a very important role in childbirth in Thailand. When a pregnant woman is in labor, they expressing a lot of pain. The traditional midwife will go ahead and deliver their clients babies people at home [5]. The midwife instructor needs to be aware of the midwives level of intelligence and the client's culture and needs to evaluate how professional care complements the mothers' traditional beliefs correlates with humanized care in the learning process in obstetrics field. Therefore, the purpose of this article is to analyze the folk medical wisdom of Thai Traditional Midwives in the South western region of Thailand.

\section{METHOD}

This study used a qualitative case studies. The qualitative research is an exploration of the perspectives and life world of human being and the meanings they give to their experience and the goal is to develop rich understanding of a phenomenon in the real world of individual perspectives. [6] Additionally, a method that allows the researcher to explore a rich description of knowledge, thoughts, or concerns relating the maternal and child caring experiences. This approach aims to determine and collect the local wisdom of Thai Traditional Midwives in Thailand. Purposive and snowball sampling were used to recruite 8 Thai Traditional Midwives who met the following criteria: (a) They delivered babies on more than 10 accounts, (b) identified themselves as health care providers as "Moh Tum Yae" (c) expressed a willingness to participate in the study.

\section{A. Study Participants}

The study was approved by the Ethics Committee of Prachomklao college of nursing. The researcher contacted the head of the community in each village to obtain permission for access to Moh tum yae. The participants were identified from each District in Phetchaburi Province in Thailand. After the participants had decided to participate, the researcher contacted them to set up an interview at their homes.

All participants were free to decide whether to participate. This process had 2 phases; Firstly, a semi-structured indepthinterview was conducted based on a review relating to the aim of this study. The interview guide was developed by the researcher who validated the quality of the question by five experts in the field of qualitative research and midwifery. 
Secondly, the face-to-face interviews were conductedbetweenthe participants and their clients from the focus group. The length of the interviews varied depending on the participants' responses. On average, took one to two hours to carry out the interview in the Thai language. Moreover, to ensure confidentiality, permission for tape recording was obtained from the participants. A recorder was used to record the interviews for verbatim transcription and detailed analysis. Field notes were written to record situations observations, and critical insights that occurred during the interview. These were kept in a safe place. The findings and interpretations were reported anonymous, and their names were not label on the recorder or transcribed documents. Each participant was assigned a numbered code and their identifying information was removed from the interviews [7].

Eight Thai Traditional Midwives were interviewed in this study. All of them were middle aged and married females. Their occupations were many in agriculture and lived in rural districts and very few practiced midwifery. Their midwifery knowledge was passed on from their grandmothers, mothers and other female relatives. Two Thai traditional midwives attended some midwifery training course organized bya public sector. Most assumed their midwifery role upon the death or retirement of their grandmother, mother, or other mentor. On average they had low incomes but generally held high prestige in their communities. All participants identified themselves as Thai Buddhists and Thai traditional midwives. The traditional midwife had very high credibility about birth delivery in the area that faraway from the hospital or the health care center.Data was collected from semi structured -in depth interviews. Participants were asked the following questions "Could you please tell me about your midwifery wisdom?", "How do you know about midwifery wisdom?" The suitable probing questions were used to gain more in depth data such as the following questions"How do you feelabout labor and child care?", "What do you mean?"and"How did you respond to the situation?".

\section{DATA ANALYSIS}

Data analysis is an important step in the research process. This study was based on manifest content analysis for analyzing data and divided into several units; condense meaning unit, codes, subcategories, categories and theme [8].

\section{RESUlTS}

Two thematic meanings of experiences of folk medical Thai midwifery wisdom in the Phetchaburi Province in Thailand emerged from the data. They were "Belief in caring mothers and infants" and "Thai midwifery ceremonies".The quotations from the participants' believed and practicedin experiences of caring mother and childwere used in parenthesis: 1-8.

\section{A. Belief in Caring Mothers and Infants}

The first theme, "Beliefin caring mothers and infants,i.e.antenatal period, at birth period and postpartum period" was represented by two categories: "a religious ceremonies" and "regarding to holy objectsand spirits.

Religious ceremonies: It was said that this particular aspect of religious belief was prevalent in Thailand. The beliefs of Traditional midwives are strongly influence by Buddhism, Brahmanism and Spirits..They are in respect to a religious precept including the five commandments of the Buddha, i.e. the first panatibat, second atinatan, third adultery, fourth falsehood and fifth liqoure mirsemashko, the rules of base metal who believed in sins and retribution. Buddhist prayed and meditation practice is used for pregnant women to gain strength in both body and mind. As one participant said:

"I always told the Buddhism pregnant women to kept in the five commandments of the Buddha .I believed that killing animal was completely immoral. The pregnant woman always said true. There did not take drugs and narcotics, not gambling and prohibited the killing. Moreover, Thai Buddhist believed that putting foods in the bowl of priest every morning that make the day was great because it was a good start at all. Furthermore, I was praying and concentrated meditation before bed. Do the right thing wil lprotected us from all obstacles, problems, difficulties and the good thing that will come to life." (2)

In regards to holy object sand spirits: this article shows that Thai Traditional Midwives had faith in the holy objects and spirits:

"Ancestral spirits and spirits heroes were protected both mothers and newborn babies, who believed in their. I always had a home or a rustic home. (Heart or center of the house) with a symbol that was believed holy. All of them will protect and help easier delivered their babies." (1)

"My knowledge passed on the from both my grandmother and nun(Mae She Noi) who taught me to delivered the pregnant and use magic that call Katar Dub Pit Fai) for protected the mother from fire in Yu- fai period." (2)

"The pregnant woman took a rest after meals, walked to exercise, massaged their legs, arms, back and belly to relive muscles and use a lot of herbs in cooking. We believed that to give birth easily." (3)

"After birth the ancient people believed that the heat from fire to warm the body, relief pain and fatigue. Moreover, the hot herbal water would kept warm temperature rises and improved blood circulation." (5)

"After cutting the umbilical cord and showers baby already. I brought the baby to put on a bamboo basket. Just then raised the basket and rotated it to the right 3 times and say "One of the ghost four children who had been taken too" This protected the baby from evil spirits." (8)

The religious beliefs of Buddhism, Brahmanism and Spirits were mixed. It conveys a sense of the social relations of pregnant women into a relationship with the divine power over nature and people. So The beliefs influence childbirth and human life.

\section{B. Ceremony for Practiced with Pregnant Women}

Participants described traditional midwifery action for caring pregnant woman in three aspects. There were composed of antenatal period, on the point of delivery period and postnatal period.

Thai midwifery in antenatal period ceremonies: Regarding antenatal care Thai traditional midwives who take care of a pregnant women by performing "Kud-thong massage" ( massages that soothe the tension of delivery by rubbing gently the belly). As participants described: 
"Kud-thong massage was a method to help moved the baby in the womb of seven months of pregnancy period. The method were; Firstly, The pregnant woman lying in a comfortable position. After that, I sit next to and used my thumb and forefinger to feel the pubis to the uterine. Secondly, I use my heel of hand, slowly push up bottom baby. The mother felt better comfortable when the babies move. Lastly, I inserted under the fingertips under baby's hand and ribs spread gradually moved my hand to the baby's head gently." (3)

"Kud-thong massage had many useful that was to said help child move from pressed on the bladder, reduce urinary symptoms, improve the blood circulation and reduce back pain." (1)

The traditional midwife takes care of a pregnant woman by examining the baby externally while it is in the womb. Midwives give massages for relaxation on the abdomen to correct the position of the baby if necessary. They also give advice on proper diet (especially herbal food) and on nourishing the mother the so that she will have an easy delivery.

At birth: The role of the traditional midwives addition of services delivery the baby such as massages, herbal medication, magical or religious ceremonies, birth care and advice. As was related below:

"I put it to use various tools to bring the water to boil. Wash my hands clean. I will support the mother, massage with hot herbal compress, shovel the stomach. After that. I gave birth by supported baby's head and pulled its. After birth, I took care of the baby before it reaches the mouth of mucus and makes breathing produced. Until then, I cut the umbilical cord with a piece of bamboo and cleaned blood and lipid coating by apply coconut oil. Clean it. So the baby to lay on the couch and prepared to care for a baby when it comes out and I will take care of her. Presses and shovel the uterus for delivered the placenta from the mother." $(1,3,4,7$, 8)

"First of all, I cleaned the vaginal area, examined the opening of cervix, helps deliver the baby, uses some herbs to prevent tearing of the perineum, cuts the cord, and performs ritual while burying the cord." $(2,3,4,8)$

If the placenta retained after birth for more than thirty minutes, all of participants used some type of technique to help their clients:

"I really know that the mother will died if the placenta retained in the uterus. If saw that I will take roll herbal leaf in her mouth and when she vomited the placenta will delivered." $(4,7,8)$

"Afterbirth I caught hold of umbilical cord due to protect its may be returned to the uterus (called "Broken Wing") and caused many mother died. This was difficulty step of placenta birth." $(4,8)$

Postnatal period ceremonies: The majority of Thai mothers who adhered to traditional postpartum practices were able to relate to the notion of regaining heat. These practices include lying by fire, taking herbal bath, consuming hot herbal drinks, breast milk care, relaxing massage, herbal compresses, sitting above charcoal, heated salt-filled pot compresses and abdominal wrapping. As was related below:

"The mother was very tired after birth. Therefore, They must be taken care .In the past, after birth mother must be lying in the fire by side on a wooden singles bed. I told clients clamp their legs for healing the perineum wound. The period in this step no less than 3 days." (4)

"Postpartum women often bloody and fishy smells so bath was required to remove stains and smell herbs boiled with water. There is commonly used herbal such as tamarind leaves ,lemon grass and onions with equal amount The hot water will help keep the pores open. Since the opening of the herbs can be absorbed through the skin into the subcutaneous will felt refreshed. It also helps deodorize amniotic fluid. And help the body get rid of impurities." (5)

"I practiced massage for treatment, improved the blood circulation, motivate the body movement smoothly in accordance to the normal function and Relieve pain of the woman who just delivered." (6)

"Herbal compress was another Thai traditional physical treatment. I used the fresh herbs i.e.: Plai, KhaminChan, Takrai,Ma Grud, Ma Kham, Som Poi and fresh pepper were mixed and wrapped in a small piece of cloth as a round chunk. By making hot compresses at the parts of body (the woman after being delivered of a baby). Herbal compress could relieve the joints and muscle sprains, initially relieve the muscle inflammation developed with 1-2 days." (6)

\section{DISCUSSION}

The experiences in caring for mothers and infants of Thai midwifery in the Phetchaburi Province in Thailand depended on their level of knowledge. From the major finding; Firstly, they were beliefs that by caring for mothers and infants (i.e. antenatal, at birth and postpartum belief"') was associatedwith religious ceremoniesand in regards to holy objects and ghosts.It was said that this particular aspect of religious belief was prevalent in Thailand. The belief of Traditional midwives is strongly influenced by Buddhism, Brahmanism and Ghosts. They believe in sins and retribution. They advise their clients to pray and meditate to gain strength in both body and mind. It conveys a sense of social relations within pregnant women into a relationship with the divine power over nature. The belief can influence childbirth and human life. The credible health care workers are those who apply morality and ethical behavior to their lives and have inner peacefulness. This reflects a deep understanding and integration of Buddhist doctrine in their daily lives. In accordance with the Buddhist teachings, as noted by Hanh and Khanthong [9], [10].People who have an understanding of the nature of things will be able to solve their own problems, transform their suffering and have more joy, peace and love. The essence of Buddhism is not to focus on the supernatural miracles and supernatural powers, despite emphasis on the moral and spiritual purification. .

Secondly, Thai traditional midwifery ceremonies were show in three aspects. First, Thai midwifery in Antenatal period ceremonies. The traditional midwife take care of a pregnant woman by examining the baby internal while it is in the womb, giving massages for relaxation, giving advice on proper diet(especially herbal food) and on nourishing the mother so that she will have easy delivery[10], [11]. Secondly, The role of the traditional midwives provide many related services in addition to birth delivery, such as massages, herbal medication, religious ceremonies, birth care 
and advice. [11], [12]. Lastly, The majority of Thai mothers adhered to the traditional postpartum period. This included lying by fire, taking herbal bath, consuming hot herbal drinks, breast milk care, relaxing massage, herbal compresses, sitting above charcoal, heated salt-filled pot compresses and abdominal wrapping. [9], [10], [13]. These national findings of the Thai midwives became an important aspect of Thai traditional medicine and also has been passed on and accepted from the past to the present.

\section{CONCLUSION AND RECOMMENDATION}

In conclusion, as the demographic background of the participants, it was found that all of them were religious people who practiced Buddhism in their daily lives so they can understand the nature of truth and live with happiness. According to the Thai traditional midwives views, the obstetric ceremonies are composed ofreligious belief and to holy objects and ghosts. In regards the significance of Thai traditional wisdom in practices ceremonies for maternal and infant care all of steps. The result of this study added to the existing knowledge about the wisdom of Thai Traditional midwifery knowledge within the context of Thai culture. This additional information could improve obstetrics in nursing and research. For example, the knowledge gleaned from the study could so help both clients and nursing students gain knowledge of midwifery by fostering their ability to obtain an understanding of the natural childbirth. Future research is needed in order to consider the development of model wisdom midwifery. Replication of this study would be advisable using midwives from different ethnic backgrounds and religious preferences.

Regarding the limitation of this study, one must use caution in generalizing the findings of Thai midwives in other socio-cultural setting. In addition, since this was a qualitative research, one has to assume that the participants were truthful with the primary researcher during the interview process.

\section{ACKNOWLEDGMENT}

We are grateful for the Thai traditional midwives who participated in this study and Prachomklao College of Nursing, Petchaburi province, Thailand, for providing financial support.

\section{REFERENCES}

[1] S. Jirojwong, "Health beliefs and the use of antenatal care among pregnant women in southern Thailand," in P. L. Rice, L. Manderson editor, Maternity and Reproductive Health in Asian Societies, Amsterdam: Harwood Academic Publisher, 1996.

[2] W. R. E. Spirituality, The 7 Central Practices to Awaken Heart and Mind, New York, John Wiley \&Sons, 1999.

[3] Buddhadasa. (2012, May 10). The Threshold Training. [Online]. Avialable: http://www.Wfb.hg.org/Buddhism.htm

[4] P. A. Payutto, Dependent Origination: The Buddhist Law of Conditionality, $3^{\text {rd }}$ ed, Bangkok: Sahathammik, 1999.

[5] A. Kansukcharearn, "Thai traditional," Midwiferyin Obstetrics Ward Report Petchburi, Petchburi Province, 2011.

[6] I. Hollowayand and S. Wheeley, Qualitative Research for Nurses, ${ }^{\text {3nd }}$ ed. United Kingdom, 2010.

[7] Forum for Ethical Review Committees in Thailand. (March 19, 2012). International Ethical Guidelines for Biomedical Research Involving Human Subject. [Online]. Available: http://www.fercit.org/ihrp/CIOMS_thai.pdf

[8] U. H. Graneheim and B. Lundman, "Qualitative content analysis in nursing research: concepts, procedures and measures to achieve trustworthiness," Nurse Education Today, vol. 24, pp. 105-112, 2004.

[9] T. N. Hanh, Master the Five Precepts for Better Governance. The Nation, 29 May, 2007, Sect. A : 3.

[10] T. Zen Khanthong, Master Rekindle our Consciousness, The Nation, vol. 1, no. 4, May 2007.

[11] P. Kaewsarn, W. Moyle, amd D. Creedy, "Traditional postpartum practices among Thai women," Journal of Advance Nursing, vol. 41, pp. $358-366,2003$

[12] S. Pornbunditpattama, "Folk Wisdom of Muslim Midwives (Tohbidae Practing in the Five Southern-Border Provinces of Thailand," Journal of Thai Traditional and alternative medicine, vol. 5, no. 3, pp. 237-241, 2007.

[13] S. Kitsripisarn et al., Perspective on Wisdom-Health of Thai Buddhist Nurses through Meditation, Thai journal nursing research, vol. 12, no. 4, pp. 297-309, 2008.

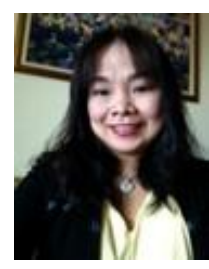

Angsinee Kansukcharearn was born in Uttaradit Province, Thailand, on 16 th Febuary 1971. She gained a bachelor degree of Nursing Science, from Prachomklao College of Nursing, Petchaburi, Thailand in 1993 and a master degree of Educational Technology from Srinakarinwirot University, Thailand, 2001. She is currently a senior lecturer in Obstetrics Nursing science at Department of midwifery Prachomklao College of nursing Petchaburi Province, Thailand. Her favorite publication is one that identifies the result of relief post casarean section pain in mothers "Developing a Pain Management Model for Women Who Underwent Cesarean Section in Prachomklao Hospital, Thailand" this will shortly appeared in The COGI proceeding book in 2012 . Her current research interests focus on Maternal and Child health, Adolescence lifestyle and Educational technology. 